BMJ Open Sport \& Exercise Medicine

\section{Effect of knee arthroplasty on sports participation and activity levels: a systematic review and meta-analysis}

To cite: Konings MJ, De Vroey H, Weygers I, et al. Effect of knee arthroplasty on sports participation and activity levels: a systematic review and meta-analysis. BMJ Open Sport \& Exercise Medicine 2020;6:e000729. doi:10.1136/ bmjsem-2019-000729

- Additional material is published online only. To view please visit the journal online (http://dx.doi.org/10.1136/ bmjsem-2019-000729).

Accepted 3 May 2020

\section{SLinked}

- http://dx.doi.org/10.1136/ bmjsem-2020-000822

\section{Check for updates}

(C) Author(s) (or their employer(s)) 2020. Re-use permitted under CC BY-NC. No commercial re-use. See rights and permissions. Published by BMJ

${ }^{1}$ Department of Rehabilitation Sciences, KU Leuven Campus Bruges, Brugge, Belgium ${ }^{2}$ Department of Rehabilitation Sciences, KU Leuven, Leuven, Flanders, Belgium

Correspondence to Dr Marco J Konings; marco.konings@kuleuven.be

\section{ABSTRACT}

Objective Desires and expectations of patients in regard to resume participation in sport activities after knee arthroplasty strongly increased in recent years. Therefore, this review systematically reviewed the available scientific literature on the effect of knee arthroplasty on sports participation and activity levels.

Design Systematic review and meta-analysis. Data sources PubMed, Embase, SPORTDiscus and reference lists were searched in February 2019. Studies eligibility criteria Inclusion of knee osteoarthritis patients who underwent total knee arthroplasty (TKA) and/or unicondylar knee arthroplasty. Studies had to include at least one preoperative and one postoperative measure ( $\geq 1$ year post surgery) of an outcome variable of interest (ie, activity level: University of California, Los Angeles and/or Lower Extremity Activity Scale; sport participation: type of sport activity survey). Results Nineteen studies were included, consisting data from 4074 patients. Knee arthroplasty has in general a positive effect on activity level and sport participation. Most patients who have stopped participating in sport activities in the year prior to surgery, however, do not seem to reinitiate their sport activities after surgery, in particular after a TKA. In contrast, patients who continue to participate in sport activities until surgery appear to become even more active in low-impact and mediumimpact sports than before the onset of restricting symptoms.

Conclusions Knee arthroplasty is an effective treatment in resuming sports participation and physical activity levels. However, to achieve the full benefits from knee arthroplasty, strategies and guidelines aimed to keep patients capable and motivated to participate in (lowimpact or medium-impact) sport activities until close before surgery are warranted.

\section{INTRODUCTION}

Knee arthroplasty (KA) is a well-accepted surgical procedure for end-stage knee osteoarthritis aiming to relieve pain, to restore normal knee function and to improve quality of life. ${ }^{12}$ Both unicondylar knee arthroplasty (UKA) and total knee arthroplasty (TKA) procedures showcase good to excellent results based on clinical testing and patientreported outcome measures. ${ }^{2}$ In recent years,

\section{What is already known?}

- Knee arthroplasty is a well-accepted surgical procedure for end-stage knee osteoarthritis aiming to relieve pain, to restore normal knee function and to improve quality of life.

- Desires and expectations of patients in regard to resume participation in sport activities after knee arthroplasty strongly increased in recent years.

\section{What are the new findings?}

Knee arthroplasty is an effective treatment in resuming sports participation and physical activity level, in particular in case patients remain active in lowimpact to medium-impact sports until close prior to surgery.

- To achieve the full benefits from knee arthroplasty, strategies and guidelines aimed to keep patients capable and motivated to participate in (low-impact or medium-impact) sport activities until close before surgery are warranted.

UKA procedures are gaining popularity in the management of unicompartmental knee osteoarthritis in comparison to TKA. ${ }^{3}{ }^{4} \mathrm{~A}$ UKA only replaces the compartment (medial or lateral) that demonstrates the most degenerative lesions and preserves both cruciate ligaments, which is believed to be beneficial for joint stability and proprioception. ${ }^{5}$ UKA has been associated with less postoperative complications and a shorter hospital stay compared with TKA, ${ }^{6-8}$ although UKA implants are more frequently revised. ${ }^{9}$

Last years, the number of KA procedures in western countries strongly increased, in particularly for relatively younger and more active individuals. ${ }^{10} 11$ This trend leads to increased desires and expectations of patients in regard to continued participation in sports activities after KA. ${ }^{12} 13$ There is wide consensus that regular exercise is essential for healthy ageing and offers many health benefits, including beneficial effects on the cardiovascular system, muscle strength, 
coordination, balance and general well-being, ${ }^{14-16}$ and reduced risk of all-cause mortality. ${ }^{17}$ In this respect, it is known that there are complex factors that affect participation in physical activity after KA such as knee function, personal barriers and beliefs, self-efficacy, social support and ageing. ${ }^{18}$ Recommendations regarding participation in a particular sport after KA are currently however still mainly based on expert opinions rather than on scientific foundation. ${ }^{12} 131519-24$

There has been considerable debate about the potentially negative long-term effects of participation in sport activities on prosthetic wear, loosening and revision rates, ${ }^{13-16}{ }^{19-21}$ despite evidence for this being rather limited..$^{25} 26$ The potential negative complications of returning to sports should be considered in balance to the beneficial effects of exercise as stated above. ${ }^{14-16}$ Patients who participated in sport following KA were found, for example, to have significantly better postoperative knee scores and lower body mass index when compared with inactive patients, although there is large variation. ${ }^{27-32}$ Pietschmann $e t a l^{27}$ even found that active patients tended to have less pain after surgery, although others have not reported such a relation between reported pain relief and number of sport activities. ${ }^{33-35}$ Importantly though, patients who become involved in sports activities after knee surgery are in general more satisfied with the outcome of the surgery, 2728333637 among the perceived facilitators and barriers to exercise after surgery, reasons not related to the replaced knee are reported more frequently than those related to the replaced knee. ${ }^{27} 3337$ As such, an increasing number of experts propagates nowadays for increasing activity after $\mathrm{KA}$, excluding high impact and/or contact sports.

The incline in surgical procedures, the increased desires and expectations of patients to continue participation in sports activities, and the well-established positive impact of exercise on experienced quality of life, urge the need for evidence-based guidelines on sport participation after KA. As a first step to improve our insights on this topic, the present review aimed to systematically evaluate the available scientific literature on the effect of KA procedures on sports participation and activity levels after the rehabilitation period. Three research questions have been formulated in order to fully elucidate the aim of this review: (1) how does sports participation and activity level change from 1 year prior to knee replacement surgery (Pre-KA) to at least more than 1 year post-knee arthroplasty (Post-KA)? (2) How does sports participation and activity level change from before the onset of restricting symptoms (Pre-ORS) to within 1 year presurgery (Pre-KA)? (3) How does sports participation and activity level change from Pre-ORS to at least more than 1 year Post-KA? For each research question, we will look into the effect of KA in general, as well as evaluate the effect of UKA and TKA procedures in specific, on sports participation and activity levels. We hypothesise KA will have a positive effect on sports participation and activity levels compared with the situation from within 1-year presurgery, in particular in patients who remain active until close before surgery. In comparison to Pre-ORS, we expect participation in low-impact sports to increase after KA, while we expect participation in high-impact sports to decrease. In addition, we expect sport participation to be higher after UKA in comparison to TKA.

\section{METHODS}

This review was conducted according to the Preferred Reporting Items for Systematic Reviews andMeta-Analyses guidelines. $^{38}$

\section{Search strategy}

The electronic databases of PubMed, Embase and SPORTDiscus were searched for relevant literature published after 1 January 2000. Searches were performed until 15 February 2019. In all three databases, the following two categories of keywords (and related synonyms) were used to build a sensitive, systemic search strategy: (1) 'knee arthroplasty', (2) 'sports' and/or 'activity'. In PubMed, we strived to use medical subject headings; otherwise, we searched the title, or title and/or abstract. Furthermore, search terms were truncated through the use of a * symbol in order to find all terms beginning with a specific word. The exact details of the search strategy can be found in online supplementary appendix A. Results were filtered for retrieval of only studies which used human participants.

\section{Inclusion criteria and study selection}

After removal of duplicates, the first author (MJK) screened all entries by both title and abstract. Hereafter, the first author (MJK) scanned the full-text of all eligible studies against the inclusion and exclusion criteria. In addition, the reference lists of selected articles were screened to identify additional articles to be included. Inclusion criteria were (1) patients with knee osteoarthritis who underwent TKA and/or UKA; who (2) intended to initiate or resume any level of sport activity after surgery; and (3) studies that included at least one preoperative and one postoperative measure ( $\geq 1$ year post surgery) of an outcome variable of interest. The outcome variable of interest to assess sport activity levels was University of California, Los Angeles (UCLA) rating scores. Studies using Lower Extremity Activity Scale (LEAS) scores were included as well, as LEAS scores can be converted into UCLA scores as described in Ghomrawi $e t a l .{ }^{39}$ Type of sport activity surveys, describing the number of participants active in a certain sport activity at a particular point in time, were used as the outcome variable of interest to assess sports participation. To ensure postoperative sports participation and activity levels are not confounded by knee functional scores, at least one postoperative measure needed to occur 1 year or longer after the date of surgery. This is because evidence suggests that the greater part of the knee function will have been regained at 1 year after surgery. ${ }^{40-42}$ Furthermore, (4) only original investigation studies (ie, no conference 
abstracts, review papers, editorials, etc), and (5) studies written in English were included.

\section{Methodological quality}

The first author (MJK) assessed the methodological quality of the included studies using the Methodological Index for Non-Randomized Studies $\left(\mathrm{MINORS}^{43}\right)$. This quality assessment method contains eight items being for both comparative and non-comparative studies, and four additional items in the case of comparative studies. MINORS has been shown to be a valid and reliable instrument designed to assess the methodological quality of non-randomised surgical studies.

\section{Data extraction}

The first author (MJK) extracted data from all selected original articles. A standardised data extraction form was used including the following topics: (1) study information: authors, year and reference number; (2) study design and type of data collection; (3) patient characteristics: sex, age, type of surgery, follow-up period; (4) preoperative and postoperative sports participation data (yes/no); (5) preoperative and postoperative activity level data (UCLA or LEAS; yes/no); (6) study biases; (7) follow-up losses/non-responders.

\section{Pooling data}

From the studies that described preoperative and postoperative participation in specific types of sport activities, data were pooled and categorised into low-impact, intermediate-impact or high-impact sport activities, according to the levels of impact on the knee joint ${ }^{13} 1920$ (see tables 1-3). Sports participation data were evaluated in respect to the number of sports per participant, and to number of sports per active participant, in which active participant is defined as any individual who was active in one or more sport activities at that particular point in time. This additional analysis of number of sports per active participant has been done to gain further insight into whether possible effects in sport activity participation are due to active patients changing their number of involved sports, inactive patients starting to participate in any sport activities, or active patients quitting completely with participation in any sport activities.

From the studies that described preoperative and postoperative sport activity levels using the UCLA and/or LEAS scales, data were pooled and a meta-analysis has been performed to determine the effect of knee replacement surgery on activity level more than 1 year after surgery expressed as change in UCLA score.

\section{RESULTS}

\section{Study selection}

The initial search identified 867 studies. A flow chart of the study selection process can be found in online supplementary appendix B. Ultimately, 19 studies were selected for the systematic review, consisting of data from in total 4074 patients. ${ }^{27-31} 3334363744-53$ Twelve studies reported data preoperatively and postoperatively on activity levels (2899 patients $293334363744-4648495152$ ), while 15 studies reported data on sport participation (2007 patients $^{27-31} 333746-53$ ). Detailed information about each included study can be found in table 4 .

\section{Methodological quality}

The methodological quality of the included studies, scored by using MINORS, ${ }^{43}$ can be found in table 5 . The average MINORS score of the included studies was 11.9 \pm 1.5 (range: $9-15$ ) out of a maximum possible score of 16. Notable common points of concern were a lack of an a priori power analysis $(\mathrm{n}=16$ studies without power analysis; $n=2$ studies with post-hoc power analysis), potential recall bias due to retrospective collection of data ( $\mathrm{n}=11$ studies), follow-up loss of more than $5 \%$ of participants or unspecified follow-up loss ( $\mathrm{n}=12$ studies) and a potential selection bias due to the participants exclusion criteria ( $\mathrm{n}=3$ studies).

\section{Participation in sport activities}

Pre-KA versus Post-KA

Eight studies examined the number of sport activities of in total 873 participants from Pre-KA to Post-KA. ${ }^{30} 33474850-53$ An overview of the participation in sport activities by the participants Pre-KA and Post-KA can be found in table 1. The change in mean number of sport activities of active participants from Pre-KA to Post-KA showed a quite similar pattern as the change in mean number of sport activities for all participants (see table 1).

\section{Pre-TKA versus Post-TKA}

Out of the eight studies, five focused on only patients who had a TKA (741 patients ${ }^{13} 37434547$ ), while Ho et a $\check{l}^{2}$ had a mixed group. Pre-TKA $49.1 \%$ did not participate (anymore) in any kind of sport activity. Post-TKA 48.7\% did not participate in any kind of sport activity. The mean number of sport activities per participant increased from Pre-TKA to Post-TKA (mean 1.9 vs 2.4 sport activities). This increase in number of sport activities Post-TKA was found in low-impact (mean 1.2 vs 1.4 sport activities), medium-impact (mean 0.4 vs 0.6 ) and high-impact sports (mean 0.3 vs 0.4 ). Similar outcomes were found when looking at the mean number of sport activities per active participant (total: 3.7 vs 4.7 sport activities; low impact: 2.3 vs 2.8 ; medium impact: 0.8 vs 1.1 ; high impact: 0.6 vs $0.8)$.

\section{Pre-UKA versus Post-UKA}

Two studies focused on only patients with UKA (160 patients, ${ }^{19}$ ) while Ho et $a l^{36}$ had a mixed group of patients with UKA and TKA. Pre-UKA, 21.9\% of patients did not participate (anymore) in any kind of sport activity. Post-UKA, 13.8\% did not participate in any kind of sport activity. The mean number of total sport activities remained quite stable from Pre-UKA to Post-UKA (mean 2.9 vs 2.8 sport activities). A very small increase in number of sport activities Post-UKA was found in low-impact (mean 1.3 vs 1.4), and medium-impact sports (mean 
Open access

Table 1 Participation per sport activity within 1 year prior (Pre-KA) and more than 1 year after knee arthroplasty (Post-KA; $\mathrm{n}=873$ )

\begin{tabular}{|c|c|c|c|c|}
\hline & Pre-KA & Post-KA & \pm & Unit of measure \\
\hline \multirow[t]{2}{*}{ Total sport activities } & 2.1 & 2.7 & & Mean $\mathrm{N}$ activities per participant \\
\hline & 4.3 & 5.0 & & \\
\hline \multirow[t]{2}{*}{ Low-impact sport activities } & 1.3 & 1.6 & & Mean $\mathrm{N}$ activities per participant \\
\hline & 2.5 & 2.9 & & Mean $\mathrm{N}$ activities per active participant \\
\hline Swimming & 371 & 449 & +178 & $\mathrm{~N}$ participants \\
\hline Cycling & 368 & 484 & +116 & $\mathrm{~N}$ participants \\
\hline Walking & 271 & 325 & +54 & $\mathrm{~N}$ participants \\
\hline Golf & 45 & 46 & +1 & $\mathrm{~N}$ participants \\
\hline Aqua aerobic & 20 & 33 & +13 & N participants \\
\hline Fishing & 18 & 12 & -6 & $\mathrm{~N}$ participants \\
\hline Gate ball & 3 & 4 & +1 & $\mathrm{~N}$ participants \\
\hline Croquet & 2 & 1 & -1 & $\mathrm{~N}$ participants \\
\hline \multirow[t]{2}{*}{ Medium-impact sport activities } & 0.5 & 0.7 & & Mean $\mathrm{N}$ activities per participant \\
\hline & 1.0 & 1.3 & & Mean $\mathrm{N}$ activities per active participant \\
\hline Hiking/Nordic walking & 309 & 476 & +167 & $\mathrm{~N}$ participants \\
\hline Fitness/aerobics & 51 & 64 & +13 & $\mathrm{~N}$ participants \\
\hline Bowling & 24 & 20 & -4 & $\mathrm{~N}$ participants \\
\hline Cross-country skiing & 23 & 27 & +4 & $\mathrm{~N}$ participants \\
\hline Badminton & 10 & 7 & -3 & $\mathrm{~N}$ participants \\
\hline Table tennis & 5 & 3 & -2 & $\mathrm{~N}$ participants \\
\hline Rowing & 3 & 0 & -3 & $\mathrm{~N}$ participants \\
\hline \multirow[t]{2}{*}{ High-impact sport activities } & 0.4 & 0.5 & & Mean $\mathrm{N}$ activities per participant \\
\hline & 0.8 & 0.8 & & Mean $\mathrm{N}$ activities per active participant \\
\hline Skiing/snowboarding & 131 & 150 & +19 & $\mathrm{~N}$ participants \\
\hline Dancing & 91 & 117 & +26 & $\mathrm{~N}$ participants \\
\hline Running/jogging & 53 & 46 & -7 & $\mathrm{~N}$ participants \\
\hline Gymnastics & 32 & 37 & +5 & $\mathrm{~N}$ participants \\
\hline Tennis/squash & 29 & 31 & +2 & $\mathrm{~N}$ participants \\
\hline Ball sports* & 12 & 4 & -8 & $\mathrm{~N}$ participants \\
\hline Mountain climbing & 2 & 7 & +5 & N participants \\
\hline Other† & 34 & 53 & +19 & $\mathrm{~N}$ participants \\
\hline No participation in any sport activity & $50 \%$ & $46 \%$ & & $\%$ of total participants \\
\hline Participation in $\geq 1$ sport activities & $50 \%$ & $54 \%$ & & $\%$ of total participants \\
\hline
\end{tabular}

*Ball sports include: soccer, basketball, volleyball and handball.

tOther involves undefined sport activities.

0.8 vs 0.9$)$. However, the mean number of high-impact sport activities did strongly decline Post-UKA (mean 0.8 vs 0.5$)$. The mean number of sport activities per active participant did decline from Pre-UKA to Post-UKA for the total number of sport activities (mean 3.7 vs 3.2) and the number of high-impact sport activities (mean $1.0 \mathrm{vs}$ $0.6)$, but the change in mean number of low-impact sport activities (1.1 vs 1.1 ) and medium-impact sport activities remained rather similar (1.6 vs 1.6).

Pre-ORS versus Pre-KA

Three studies (in total 453 participants) examined the number of sport activities from Pre-ORS up to within
1 year Pre-KA. ${ }^{305253}$ An overview of the participation in sport activities by the participants Pre-ORS and Pre-KA can be found in table 2. The change in mean number of sport activities per active participant from Pre-ORS to Pre-KA showed a quite similar pattern as the change in mean number of sport activities for all participants, except the relatively much smaller decline from Pre-ORS to Pre-KA in low-impact sport activities (see table 2).

\section{Pre-ORS versus Pre-TKA}

Two studies focused on only patients who had a TKA (381 patients ${ }^{37}$ ), while Ho $e t a l^{36}$ had a mixed group. When looking only at patients with TKA, $5 \%$ did not participate 
Table 2 Participation per sport activity before onset of restricting symptoms (Pre-ORS) and within 1 year to surgery (Pre-KA; $\mathrm{n}=453$ )

\begin{tabular}{|c|c|c|c|c|}
\hline & Pre-ORS & Pre-KA & \pm & Unit of measure \\
\hline \multirow[t]{2}{*}{ Total sport activities } & 2.7 & 0.8 & & Mean $\mathrm{N}$ activities per participant \\
\hline & 2.9 & 1.8 & & $\begin{array}{l}\text { Mean } \mathrm{N} \text { activities per active } \\
\text { participant }\end{array}$ \\
\hline \multirow[t]{2}{*}{ Low-impact sport activities } & 1.2 & 0.5 & & $\mathrm{~N}$ activities per participant \\
\hline & 1.3 & 1.1 & & $\begin{array}{l}\text { Mean } \mathrm{N} \text { activities per active } \\
\text { participant }\end{array}$ \\
\hline Cycling & 268 & 111 & -157 & $\mathrm{~N}$ participants \\
\hline Swimming & 211 & 115 & -96 & $\mathrm{~N}$ participants \\
\hline Golf & 35 & 10 & -25 & $\mathrm{~N}$ participants \\
\hline Aqua aerobic & 16 & 12 & -4 & $\mathrm{~N}$ participants \\
\hline \multirow[t]{2}{*}{ Medium-impact sport activities } & 0.7 & 0.1 & & Mean $\mathrm{N}$ activities per participant \\
\hline & 0.8 & 0.3 & & $\begin{array}{l}\text { Mean } \mathrm{N} \text { activities per active } \\
\text { participant }\end{array}$ \\
\hline Hiking/Nordic walking & 234 & 36 & -198 & $\mathrm{~N}$ participants \\
\hline Fitness/aerobics & 48 & 13 & -35 & $\mathrm{~N}$ participants \\
\hline Cross-country skiing & 30 & 15 & -15 & $\mathrm{~N}$ participants \\
\hline Bowling & 10 & 2 & -8 & $\mathrm{~N}$ participants \\
\hline \multirow[t]{2}{*}{ High-impact sport activities } & 0.8 & 0.2 & & Mean $\mathrm{N}$ activities per participant \\
\hline & 0.9 & 0.3 & & $\begin{array}{l}\text { Mean } \mathrm{N} \text { activities per active } \\
\text { participant }\end{array}$ \\
\hline Skiing/snowboarding & 121 & 17 & -104 & $\mathrm{~N}$ participants \\
\hline Gymnastics & 74 & 24 & -50 & $\mathrm{~N}$ participants \\
\hline Dancing & 69 & 18 & -51 & $\mathrm{~N}$ participants \\
\hline Running/jogging & 55 & 4 & -51 & $\mathrm{~N}$ participants \\
\hline Ball sports* & 43 & 5 & -38 & $\mathrm{~N}$ participants \\
\hline Tennis/squash & 14 & 2 & -12 & $\mathrm{~N}$ participants \\
\hline Mountain climbing & 3 & 1 & -2 & $\mathrm{~N}$ participants \\
\hline No participation in any sport activity & $7 \%$ & $51 \%$ & & $\%$ of total participants \\
\hline Participation in $\geq 1$ sport activities & $93 \%$ & $49 \%$ & & $\%$ of total participants \\
\hline
\end{tabular}

*Ball sports include: soccer, basketball, volleyball and handball.

Pre-ORS in one or more sport activities. Pre-TKA, 52\% did not participate in any kind of sport activity. The mean number of sport activities per patient with TKA strongly declines from Pre-ORS to Pre-TKA (mean 2.9 vs 0.9 sport activities). This decline in number of sport activities PreTKA can be seen in low-impact (mean 1.2 vs 0.6 sport activities), medium-impact (mean 0.8 vs 0.2 ) and highimpact sports (mean 0.9 vs 0.2 ). Even when adjusting sport activities to only active participants, a decline has been found in all types of sport from Pre-ORS to Pre-TKA (total: 3.0 vs 1.9 sport activities; low impact: 1.3 vs 1.2 ; medium impact: 0.8 vs 0.3 ; high impact: 1.0 vs 0.3 ).

Pre-ORS versus Pre-UKA

No study looked into the change in number of sport activities from Pre-ORS to Pre-UKA for patients with UKA exclusively.
Pre-ORS versus Post-KA

Ten studies examined the number of sport activities of in total 1134 participants from Pre-ORS up to at least more than a year Post-KA. ${ }^{27-31} 3746495253$ An overview of the participation in sport activities by the participants PreORS and Post-KA can be found in table 3 .

The participation in number of sport activities of active participants showed a rather different pattern as the total group of participants. The mean number of sport activities showed only a relatively small decline Post-KA versus Pre-ORS in active participants (3.0 vs 2.8 sport activities), while an increase in the mean number of low-impact $(1.2$ vs 1.4$)$ and medium-impact sport activities (0.8 vs 0.9$)$ per active participant has been found from Pre-ORS to Post-KA. Only the mean number of high-impact activities per active participant showed a strong decline Post-KA in comparison to Pre-ORS (1.0 vs 0.5 ). 
Table 3 Participation per sport activity before onset of restricting symptoms (Pre-ORS) and after knee arthroplasty (Post-KA; $\mathrm{n}=1134$ )

\begin{tabular}{|c|c|c|c|c|}
\hline & Pre-ORS & Post-KA & \pm & Unit of measure \\
\hline \multirow[t]{2}{*}{ Total sport activities } & 2.6 & 1.8 & & Mean $\mathrm{N}$ activities per participant \\
\hline & 3.0 & 2.8 & & Mean $\mathrm{N}$ activities per active participant \\
\hline \multirow[t]{2}{*}{ Low-impact sport activities } & 1.0 & 0.9 & & $\mathrm{~N}$ activities per participant \\
\hline & 1.2 & 1.4 & & Mean $\mathrm{N}$ activities per active participant \\
\hline Cycling & 588 & 463 & -125 & $\mathrm{~N}$ participants \\
\hline Swimming & 382 & 347 & -35 & $\mathrm{~N}$ participants \\
\hline Walking & 114 & 115 & +1 & N participants \\
\hline Golf & 61 & 56 & -5 & $\mathrm{~N}$ participants \\
\hline Aqua aerobic & 33 & 53 & +20 & $\mathrm{~N}$ participants \\
\hline Bowls & 3 & 3 & 0 & $\mathrm{~N}$ participants \\
\hline Fishing & 2 & 2 & 0 & $\mathrm{~N}$ participants \\
\hline Shooting & 1 & 1 & 0 & $\mathrm{~N}$ participants \\
\hline \multirow[t]{2}{*}{ Medium-impact sport activities } & 0.7 & 0.6 & & Mean $\mathrm{N}$ activities per participant \\
\hline & 0.8 & 0.9 & & Mean $\mathrm{N}$ activities per active participant \\
\hline Hiking/Nordic walking & 577 & 454 & -123 & $\mathrm{~N}$ participants \\
\hline Fitness/aerobics & 105 & 132 & +27 & $\mathrm{~N}$ participants \\
\hline Cross-country skiing & 98 & 37 & -61 & $\mathrm{~N}$ participants \\
\hline Bowling & 19 & 11 & -8 & N participants \\
\hline Badminton & 18 & 8 & -10 & N participants \\
\hline Horse riding & 3 & 0 & -3 & $\mathrm{~N}$ participants \\
\hline Table tennis & 1 & 1 & 0 & N participants \\
\hline \multirow[t]{2}{*}{ High-impact sport activities } & 0.9 & 0.3 & & Mean $\mathrm{N}$ activities per participant \\
\hline & 1.0 & 0.5 & & Mean $\mathrm{N}$ activities per active participant \\
\hline Skiing/snowboarding & 246 & 54 & -192 & N participants \\
\hline Gymnastics & 157 & 96 & -61 & $\mathrm{~N}$ participants \\
\hline Dancing & 143 & 93 & -50 & $\mathrm{~N}$ participants \\
\hline Running/jogging & 131 & 23 & -108 & N participants \\
\hline Ball sports* & 123 & 24 & -99 & $\mathrm{~N}$ participants \\
\hline Tennis/squash & 120 & 34 & -86 & $\mathrm{~N}$ participants \\
\hline Mountain climbing & 33 & 11 & -22 & $\mathrm{~N}$ participants \\
\hline Skating & 19 & 4 & -15 & $\mathrm{~N}$ participants \\
\hline Ice hockey & 2 & 1 & -1 & $\mathrm{~N}$ participants \\
\hline Boxing & 1 & 0 & -1 & $\mathrm{~N}$ participants \\
\hline Other† & 17 & 14 & -3 & N participants \\
\hline No participation in any sport activity & $11 \%$ & $35 \%$ & & $\%$ of total participants \\
\hline Participation in $\geq 1$ sport activities & $89 \%$ & $65 \%$ & & $\%$ of total participants \\
\hline
\end{tabular}

*Ball sports include: soccer, basketball, volleyball and handball.

†Other involves undefined sport activities.

\section{Pre-ORS versus Post-TKA}

Out of the 10 studies, 3 focused on only TKA (617 patients, ${ }^{374749}$ ) while Ho et $a l^{36}$ had a mixed group. When looking only at patients who got a TKA, 3\% did not participate in any kind of sport activity Pre-ORS. Post-TKA 44\% did not participate in any kind of sport activity. The mean number of sport activities per patient declined from Pre-ORS to Post-TKA (mean 2.9 vs 1.7 sport activities). This decline in number of sport activities Post-TKA could be seen in low-impact (mean 1.0 vs
0.8 sport activities), medium-impact (mean 0.8 vs 0.6 ) and high-impact sports (mean 1.0 vs 0.4 ). Remarkably, when looking at the number of sport activities per active participant, the total number of sport activities remained rather similar Post-TKA in comparison to Pre-ORS (total: 3.0 vs 3.0 sport activities). The mean number of low-impact (mean 1.1 vs 1.4 ) and medium-impact sport activities (mean 0.9 vs 1.0) even increased Post-TKA, while only the mean number of high-impact sport activities declined (mean 1.0 vs 0.6 ). 
Table 4 Detailed overview of included studies on study design, study population, measure of sports participation, measure of activity level, study biases and follow-up loss

\begin{tabular}{|c|c|c|c|c|c|c|}
\hline Study & Design & Study population & Sport participation & $\begin{array}{l}\text { Activity level (UCLA/ } \\
\text { LEAS) }\end{array}$ & Study biases & $\begin{array}{l}\text { Follow-up loss/non- } \\
\text { responders }\end{array}$ \\
\hline Scott et al/4 & $\begin{array}{l}\text { Retrospective } \\
\text { study } \\
\text { Prospective data } \\
\text { collection }\end{array}$ & $\begin{aligned} & 14 \text { female } \\
& \text { patients, } 16 \\
& \text { male patients } \\
& \text { Mean age: } 58 \pm 5 \\
& \text { years (range } \\
& 46-64) \\
& \text { Non-consecutive } \\
& \text { revision TKA } \\
\text { Follow-up: mean } & \text { Fon } \\
& 3.8 \pm 2.2 \text { years } \\
& \text { (range } 1-9 \text { ) }\end{aligned}$ & No & $\begin{array}{l}\text { Yes } \\
(\mathrm{UCLA})\end{array}$ & $\begin{array}{l}\text { No power } \\
\text { calculation }\end{array}$ & $\begin{array}{l}\text { No follow-up loss } \\
(0 \%)\end{array}$ \\
\hline Scott et $a l^{45}$ & $\begin{array}{l}\text { Retrospective } \\
\text { study } \\
\text { Prospective data } \\
\text { collection }\end{array}$ & $\begin{array}{l}148 \text { female } \\
\text { patients, } 141 \\
\text { male patients } \\
\text { Mean age } 59 \\
\text { years (range } \\
42-65 \text { ) } \\
\text { TKA } \\
\text { Follow-up: mean } \\
3.4 \text { years (range } \\
2-5 \text { ) }\end{array}$ & No & $\begin{array}{l}\text { Yes } \\
\text { (UCLA) }\end{array}$ & $\begin{array}{l}\text { No power } \\
\text { calculation }\end{array}$ & $\begin{array}{l}\text { No follow-up loss } \\
(0 \%)\end{array}$ \\
\hline Ponzio et $a l^{36}$ & $\begin{array}{l}\text { Retrospective } \\
\text { study } \\
\text { Prospective data } \\
\text { collection }\end{array}$ & $\begin{array}{l}1140 \text { female } \\
\text { patients, } 876 \\
\text { male patients } \\
\text { Mean age: } \\
66.3 \pm 9.0 \text { years } \\
\text { Unilateral } \\
\text { primary TKA } \\
\text { Follow-up: } \\
2 \text { years after } \\
\text { surgery }\end{array}$ & No & $\begin{array}{l}\text { Yes } \\
\text { (LEAS) }\end{array}$ & $\begin{array}{l}\text { Potential } \\
\text { selection bias: } \\
\text { participants with } \\
\text { low preoperative } \\
\text { LEAS scores } \\
\text { excluded; } \\
\text { No power } \\
\text { calculation }\end{array}$ & $\begin{array}{l}\text { Follow-up loss: } 517 \\
\text { out of } 2016(26 \%)\end{array}$ \\
\hline $\begin{array}{l}\text { Panzram et } \\
a l^{46}\end{array}$ & $\begin{array}{l}\text { Retrospective } \\
\text { study }\end{array}$ & $\begin{aligned} & 12 \text { female } \\
& \text { patients, } 15 \\
& \text { male patients } \\
& \text { Mean age: } 62.5 \\
& \text { years (range } \\
& 49-76 \text { ) } \\
& \text { Cementless } \\
& \text { medial UKA } \\
& \text { Mean follow-up: } \\
& 60 \pm 8 \text { months } \\
& \text { (range 47-69) }\end{aligned}$ & Yes & $\begin{array}{l}\text { Yes } \\
\text { (UCLA) }\end{array}$ & $\begin{array}{l}\text { Potential recall } \\
\text { bias } \\
\text { No power } \\
\text { calculation }\end{array}$ & $\begin{array}{l}\text { Follow-up loss: } 3 \text { out } \\
\text { of } 27(11 \%)\end{array}$ \\
\hline Chatterji et al ${ }^{47}$ & $\begin{array}{l}\text { Retrospective } \\
\text { study }\end{array}$ & $\begin{array}{l}80 \text { female } \\
\text { patients, } 64 \\
\text { male patients } \\
\text { Mean age: } \\
70.8 \pm 10.4 \text { years } \\
\text { TKA } \\
\text { Follow-up: } \\
\text { between } 1 \text { and } \\
2 \text { years after } \\
\text { surgery }\end{array}$ & Yes & No & $\begin{array}{l}\text { Potential recall } \\
\text { bias } \\
\text { No power } \\
\text { calculation }\end{array}$ & $\begin{array}{l}\text { Follow-up loss: } 34 \\
\text { out of } 178(19 \%)\end{array}$ \\
\hline
\end{tabular}




\begin{tabular}{|c|c|c|c|c|c|c|}
\hline Study & Design & Study population & Sport participation & $\begin{array}{l}\text { Activity level (UCLA/ } \\
\text { LEAS) }\end{array}$ & Study biases & $\begin{array}{l}\text { Follow-up loss/non- } \\
\text { responders }\end{array}$ \\
\hline Williams et $\left.a\right|^{34}$ & $\begin{array}{l}\text { Retrospective } \\
\text { study } \\
\text { Prospective data } \\
\text { collection }\end{array}$ & $\begin{aligned} & 155 \text { female, } 94 \\
& \text { male patients } \\
& \text { Mean age: } \\
& 67.5 \pm 9.9 \text { years } \\
& \text { (range 45-93) } \\
& 232 \text { TKA, } 17 \\
& \text { UKA } \\
& \text { Mean follow-up: } \\
& 12.1 \text { months; } \\
& \text { range } 11-13 .\end{aligned}$ & No & $\begin{array}{l}\text { Yes } \\
\text { (UCLA) }\end{array}$ & $\begin{array}{l}\text { No power } \\
\text { calculation }\end{array}$ & $\begin{array}{l}\text { Not reported for } \\
\text { patients with knee } \\
\text { arthroplasty }\end{array}$ \\
\hline Naal et $\left.a\right|^{28}$ & $\begin{array}{l}\text { Retrospective } \\
\text { study }\end{array}$ & $\begin{aligned} & 38 \text { female, } 45 \\
& \text { male patients } \\
& \text { Mean age: } \\
& 65.5 \pm 9.1 \text { (range } \\
& 47-83 \text { ) } \\
& \text { UKA } \\
& \text { Mean follow-up: } \\
& 18 \pm 5 \text { months } \\
& \text { (range } 12-28 \text { ) }\end{aligned}$ & Yes & No & $\begin{array}{l}\text { Potential recall } \\
\text { bias } \\
\text { No power } \\
\text { calculation }\end{array}$ & $\begin{array}{l}\text { Follow-up loss: } 19 \\
\text { out of } 102(19 \%)\end{array}$ \\
\hline Fisher et $a l^{29}$ & Prospective study & $\begin{array}{l}34 \text { female } \\
\text { patients, } 32 \\
\text { male patients } \\
\text { Mean age: } 64 \\
\text { years (range } \\
49-81 \text { ) } \\
\text { Oxford medial } \\
\text { UKA } \\
\text { Mean follow- } \\
\text { up: } 18 \text { months } \\
\text { (range 4-46) }\end{array}$ & Yes & $\begin{array}{l}\text { Yes } \\
\text { (UCLA) }\end{array}$ & $\begin{array}{l}\text { No power } \\
\text { calculation }\end{array}$ & $\begin{array}{l}\text { Follow-up loss: } 9 \text { out } \\
\text { of } 75(12 \%)\end{array}$ \\
\hline Huch et $a l^{53}$ & Prospective study & $\begin{array}{l}216 \text { female } \\
\text { patients, } 84 \\
\text { male patients } \\
\text { Mean age: } \\
66.0 \pm 6.4 \text { years } \\
\text { TKA } \\
\text { Follow-up: } \\
5 \text { years after } \\
\text { surgery }\end{array}$ & Yes & No & $\begin{array}{l}\text { No power } \\
\text { calculation }\end{array}$ & $\begin{array}{l}\text { Follow-up loss: } 89 \\
\text { out of } 389(23 \%)\end{array}$ \\
\hline
\end{tabular}

Continued 
Table 4 Continued

\begin{tabular}{|c|c|c|c|c|c|c|}
\hline Study & Design & Study population & Sport participation & $\begin{array}{l}\text { Activity level (UCLA/ } \\
\text { LEAS) }\end{array}$ & Study biases & $\begin{array}{l}\text { Follow-up loss/non- } \\
\text { responders }\end{array}$ \\
\hline $\begin{array}{l}\text { Pietschmann } \\
\text { et }\left.a\right|^{27}\end{array}$ & $\begin{array}{l}\text { Retrospective } \\
\text { study }\end{array}$ & $\begin{array}{l}74 \text { female } \\
\text { patients, } 57 \\
\text { male patients } \\
\text { Mean age: } 65.3 \\
\text { years (range } \\
44-90 \text { years) } \\
\text { Medial UKA } \\
\text { Oxford III } \\
\text { Mean follow-up: } \\
4.2 \text { years (range } \\
1-10 \text { ) }\end{array}$ & Yes & No & $\begin{array}{l}\text { Unclear if data } \\
\text { collection } \\
\text { preoperatively } \\
\text { and } \\
\text { postoperatively } \\
\text { was done at } \\
\text { same time or not; } \\
\text { No power } \\
\text { calculation }\end{array}$ & $\begin{array}{l}\text { Follow-up loss: } 38 \\
\text { out of } 169(22 \%)\end{array}$ \\
\hline Jahnke et $\left.a\right|^{51}$ & Prospective study & $\begin{array}{l}63 \text { female } \\
\text { patients, } 72 \\
\text { male patients } \\
\text { Mean age: } 63.5 \\
\text { years (range } \\
\text { 36-86) } \\
\text { Medial Oxford } \\
\text { UKA } \\
\text { Follow-up: } 5 \\
\text { years later }\end{array}$ & Yes & $\begin{array}{l}\text { Yes } \\
(\mathrm{UCLA})\end{array}$ & $\begin{array}{l}\text { No power } \\
\text { calculation }\end{array}$ & $\begin{array}{l}\text { Follow-up loss: } 24 \\
\text { out of } 159(15 \%)\end{array}$ \\
\hline Vielgut et $a l^{31}$ & $\begin{array}{l}\text { Retrospective } \\
\text { study }\end{array}$ & $\begin{array}{l}193 \text { female } \\
\text { patients, } 43 \\
\text { male patients } \\
\text { Mean age: } \\
62.7 \pm 11.4 \text { years } \\
\text { TKA } \\
\text { Follow-up: } \\
\text { minimum of } \\
10 \text { years post } \\
\text { surgery }\end{array}$ & Yes & No & $\begin{array}{l}\text { Potential recall } \\
\text { bias } \\
\text { Potential } \\
\text { selection bias: } \\
\text { participant } \\
\text { screening } \\
\text { preoperative } \\
\text { sports } \\
\text { participation; } \\
\text { Post-hoc power } \\
\text { calculation }\end{array}$ & $\begin{array}{l}\text { Follow-up loss: } 8 \text { out } \\
\text { of } 244(3 \%)\end{array}$ \\
\hline Mayr et $\left.a\right|^{30}$ & $\begin{array}{l}\text { Retrospective } \\
\text { study }\end{array}$ & $\begin{array}{l}43 \text { female, } 38 \\
\text { male patients } \\
\text { from alpine area } \\
\text { Mean age of } \\
71.8 \pm 5.4 \text { years } \\
\text { TKA } \\
\text { Mean follow-up: } \\
6.4 \pm 0.9 \text { years }\end{array}$ & Yes & No & $\begin{array}{l}\text { Potential recall } \\
\text { bias }\end{array}$ & $\begin{array}{l}\text { Follow-up loss: } 2 \text { out } \\
\text { of } 83(2 \%)\end{array}$ \\
\hline $\begin{array}{l}\text { Hepperger et } \\
\left.a\right|^{50}\end{array}$ & Prospective study & $\begin{array}{l}120 \text { female, } 80 \\
\text { male patients } \\
\text { from alpine area } \\
\text { Mean age: } \\
72.2 \pm 7.7 \text { years } \\
\text { Primary TKA } \\
\text { Follow-up: } 24 \\
\text { months post } \\
\text { surgery }\end{array}$ & Yes & No & $\begin{array}{l}\text { Post-hoc power } \\
\text { calculation }\end{array}$ & $\begin{array}{l}\text { Follow-up loss: } 3 \text { out } \\
\text { of } 203(1 \%)\end{array}$ \\
\hline Walker et al ${ }^{49}$ & $\begin{array}{l}\text { Retrospective } \\
\text { study }\end{array}$ & $\begin{array}{l}47 \text { female } \\
\text { patients, } 46 \\
\text { male patients } \\
\text { Mean age: } 55 \pm 5 \\
\text { years (range } \\
36-60 \text { ) } \\
\text { Medial UKA } \\
\text { Mean follow-up: } \\
4.4 \pm 1.6 \text { years } \\
\text { (range } 2-8 \text { ) }\end{array}$ & Yes & $\begin{array}{l}\text { Yes } \\
(\mathrm{UCLA})\end{array}$ & $\begin{array}{l}\text { Potential recall } \\
\text { bias } \\
\text { No power } \\
\text { calculation }\end{array}$ & $\begin{array}{l}\text { Follow-up loss: } 8 \text { out } \\
\text { of } 101(8 \%)\end{array}$ \\
\hline
\end{tabular}


Table 4 Continued

\begin{tabular}{|c|c|c|c|c|c|c|}
\hline Study & Design & Study population & Sport participation & $\begin{array}{l}\text { Activity level (UCLA/ } \\
\text { LEAS) }\end{array}$ & Study biases & $\begin{array}{l}\text { Follow-up loss/non- } \\
\text { responders }\end{array}$ \\
\hline Ho et $\left.a\right|^{52}$ & $\begin{array}{l}\text { Retrospective } \\
\text { study }\end{array}$ & $\begin{aligned} & 48 \text { female } \\
& \text { patients, } 24 \\
& \text { male patients } \\
& \text { Mean age } 60.0 \\
& \text { years (range } \\
& 53-64 \text { ) } \\
& 33 \text { UKA, 39 TKA } \\
& \text { Mean follow-up: } \\
& 45.6 \text { months } \\
& \text { (range 24-68) }\end{aligned}$ & Yes & $\begin{array}{l}\text { Yes } \\
\text { (UCLA) }\end{array}$ & $\begin{array}{l}\text { Potential recall } \\
\text { bias } \\
\text { No power } \\
\text { calculation }\end{array}$ & $\begin{array}{l}\text { Follow-up loss not } \\
\text { specified }\end{array}$ \\
\hline
\end{tabular}

LEAS, Lower Extremity Activity Scale; TKA, total knee arthroplasty; UCLA, University of California, Los Angeles; UKA, unicondylar knee arthroplasty.

Pre-ORS versus Post-UKA

Six studies focused on only patients who had a UKA (445 patients ${ }^{33} 3439414448$ ), while Ho $e t a l^{36}$ had a mixed group. Of the patients who got a UKA, 22\% did not participate (anymore) in any kind of sport activity PreORS. Post-UKA, 25\% did not participate in any kind of sport activity. The mean number of total sport activities per participant did decline from Pre-ORS to Post-UKA (mean 2.4 vs 2.0 sport activities), mainly due to a reduced number of high-impact sports Post-UKA (mean 0.7 vs 0.2 ). The mean number of sport activities per participant in low-impact (mean 1.1 vs 1.1) and medium-impact sports (mean 0.6 vs 0.6 ) was rather similar at Pre-ORS and Post-UKA. Comparable outcomes were found when looking at the number of sport activities per active participant (total: 3.1 vs 2.6 sport activities; low impact: 1.4 vs 1.5; medium impact: 0.8 vs 0.8 ; high impact: 0.9 vs 0.3 )

\section{Activity level}

Pre-KA versus Post-KA

Nine studies reported UCLA scores within 1 year prior to surgeryand more than 1 yearpostoperatively, ${ }^{2933343744-464849}$

Table 5 The methodological quality of the included studies scored using Methodological Index for Non-Randomized Studies

\begin{tabular}{|c|c|c|c|c|c|c|c|c|c|}
\hline Study & $\begin{array}{l}\text { 1. A } \\
\text { clearly } \\
\text { stated } \\
\text { aim }\end{array}$ & $\begin{array}{l}\text { 2. Inclusion } \\
\text { of } \\
\text { consecutive } \\
\text { patients }\end{array}$ & $\begin{array}{l}\text { 3. Prospective } \\
\text { collection of } \\
\text { data }\end{array}$ & $\begin{array}{l}\text { 4. Endpoint } \\
\text { appropriate } \\
\text { to the study } \\
\text { aim }\end{array}$ & $\begin{array}{l}\text { 5. Unbiased } \\
\text { assessment } \\
\text { of endpoints }\end{array}$ & $\begin{array}{l}\text { 6. Follow-up } \\
\text { period } \\
\text { appropriate } \\
\text { to study aim }\end{array}$ & $\begin{array}{l}\text { 7. Loss to } \\
\text { follow- } \\
\text { up not } \\
\text { exceeding } \\
5 \%\end{array}$ & $\begin{array}{l}\text { 8. Prospective } \\
\text { calculation of the } \\
\text { study size }\end{array}$ & $\begin{array}{l}\text { Total } \\
\text { score }\end{array}$ \\
\hline Chang et $a^{33}$ & 2 & 1 & 1 & 2 & 2 & 2 & 1 & 0 & $11 / 16$ \\
\hline Scott et al ${ }^{44}$ & 2 & 1 & 2 & 1 & 2 & 2 & 2 & 0 & $12 / 16$ \\
\hline Scott et al ${ }^{45}$ & 2 & 2 & 2 & 1 & 2 & 2 & 2 & 0 & $13 / 16$ \\
\hline Ponzio et al ${ }^{36}$ & 2 & 2 & 2 & 2 & 1 & 2 & 1 & 0 & $12 / 16$ \\
\hline Panzram et al ${ }^{46}$ & 2 & 2 & 1 & 2 & 2 & 2 & 1 & 0 & $12 / 16$ \\
\hline Chatterji et al ${ }^{47}$ & 2 & 2 & 1 & 2 & 2 & 2 & 1 & 0 & $12 / 16$ \\
\hline Canetti et al ${ }^{48}$ & 2 & 1 & 1 & 2 & 1 & 2 & 2 & 0 & $11 / 16$ \\
\hline Williams et al ${ }^{34}$ & 2 & 2 & 2 & 1 & 2 & 1 & 0 & 0 & $10 / 16$ \\
\hline Naal et al ${ }^{28}$ & 2 & 2 & 1 & 2 & 2 & 2 & 1 & 0 & $12 / 16$ \\
\hline Walker et al ${ }^{37}$ & 2 & 2 & 1 & 2 & 2 & 2 & 2 & 0 & $13 / 16$ \\
\hline Fisher et al ${ }^{29}$ & 2 & 2 & 2 & 2 & 2 & 1 & 1 & 0 & $12 / 16$ \\
\hline Huch et al ${ }^{53}$ & 2 & 2 & 2 & 2 & 2 & 2 & 1 & 0 & $13 / 16$ \\
\hline $\begin{array}{l}\text { Pietschmann et } \\
\text { al }{ }^{27}\end{array}$ & 2 & 2 & 0 & 1 & 2 & 2 & 1 & 0 & $10 / 16$ \\
\hline Jahnke et al ${ }^{51}$ & 2 & 0 & 2 & 2 & 2 & 2 & 1 & 0 & $11 / 16$ \\
\hline Vielgut et al ${ }^{31}$ & 2 & 1 & 1 & 2 & 1 & 2 & 2 & 1 & $12 / 16$ \\
\hline Mayr et al ${ }^{30}$ & 2 & 2 & 1 & 2 & 2 & 2 & 2 & 2 & $15 / 16$ \\
\hline $\begin{array}{l}\text { Hepperger et } \\
\text { al }\end{array}$ & 2 & 2 & 2 & 2 & 2 & 2 & 2 & 1 & $15 / 16$ \\
\hline Walker et al ${ }^{49}$ & 2 & 2 & 1 & 2 & 2 & 2 & 1 & 0 & $12 / 16$ \\
\hline Ho et al $^{52}$ & 2 & 0 & 1 & 2 & 2 & 2 & 0 & 0 & $9 / 16$ \\
\hline
\end{tabular}

*The items are scored 0 (not reported), 1 (reported but inadequate) or 2 (reported and adequate). The global ideal score being 16 for noncomparative studies. 


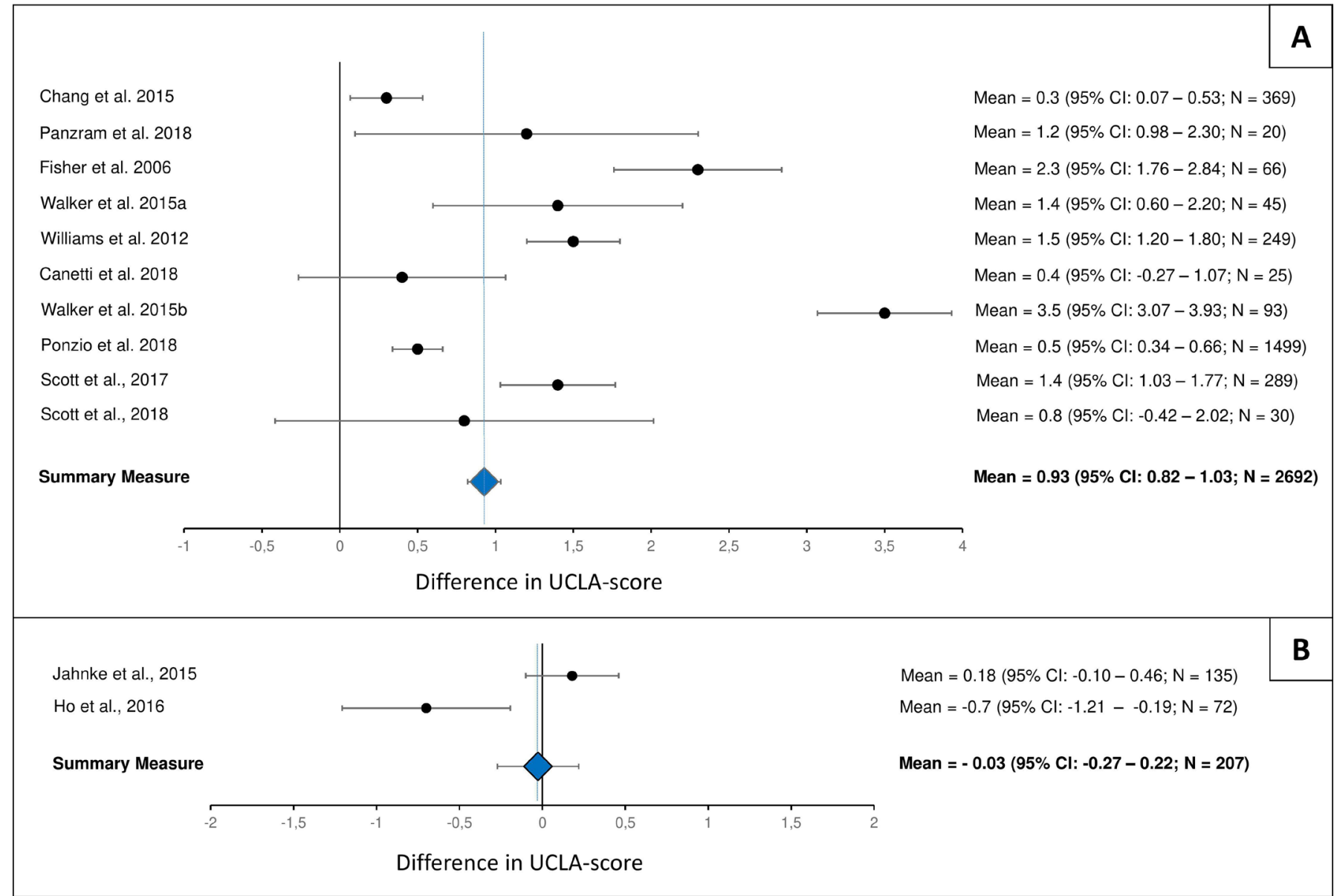

Figure 1 Meta-analysis on change in UCLA (University of California, Los Angeles) scores from within 1-year pre-knee replacement surgery to more than 1-year post-knee replacement surgery $(n=10$ studies; $N=2692$ participants; upper section A); and meta-analysis onchange in UCLA scores from pre-onset of restricting symptoms to more than 1-year post-knee replacement surgery $(n=2$ studies; $N=207$ participants; lower section $B)$.

while one study reported LEAS scores. ${ }^{36}$ The effects of KA on activity level (ie, UCLA score) relative to within 1 year pre-knee replacement surgery can be found in figure 1 . The summary measure of the meta-analysis of 2692 participants revealed that the mean UCLA score more than a year Post-KA improved in comparison to the UCLA score within 1 year prior to surgery (Pre-KA: 5.6 \pm 1.1 ; Post-KA: $6.5 \pm 1.2$ ).

Half of the 10 studies focused on patients who had an UKA, ${ }^{13} 20384042$ while the other half focused primarily on patients with TKA. ${ }^{19} 33343941$ An additional analysis revealed that the extent of the improvement in UCLA scores was higher after UKA (mean improvement in UCLA score: $2.3 \pm 0.4 ; 256$ patients), in comparison to TKA (mean improvement: $0.7 \pm 0.2 ; 2436$ patients). The Post-KA UCLA scores were almost similar regardless of type of surgery (UKA Post-KA: 6.6 \pm 1.4 ; TKA Post-KA: $6.5 \pm 2.0)$.

\section{Pre-ORS versus Pre-KA}

No studies have been found that measured the change in activity level from Pre-ORS to within the year before surgery.

\section{Pre-ORS versus Post-KA}

Two studies reported UCLA scores Pre-ORS and more than 1 year postoperatively. ${ }^{51}{ }^{52}$ The effects of KA on activity level (ie, UCLA score) relative to Pre-ORS can be found in figure 1. The summary measure of the meta-analysis of 207 participants revealed that the mean UCLA score did not differ between Pre-ORS and Pre-KA (mean $=-0.03 ; 95 \% \mathrm{CI}-0.27$ to 0.22 ). No additional analyses were performed into possible differences between UKA and TKA due to the low number of studies.

\section{DISCUSSION}

This review aimed to systematically review the scientific literature on the effect of KA on sports participation and activity levels. KA has been found to have in general a positive effect on sports participation and activity level in comparison to the situation in the year before surgery, with activity levels returning in the years after KA to a similar level as Pre-ORS. Most patients who have stopped participating in sport activities in the year prior to surgery, however, do not seem to reinitiate their sport activities after surgery, in particular in case of TKA. In contrast, patients who continued to participate in sport activities 
until surgery appear to become similarly or even more active in low-impact and medium-impact sports than Pre-ORS. Participation in high-impact sports strongly declined in comparison to Pre-ORS.

As one may expect, sport participation strongly declined from the period Pre-ORS until within 1year prior to surgery. Prior to surgery, the majority of patients reported pain or a physical limitation due to their knee osteoarthritis (eg, loss of range of motion, stiffness, swelling, inability to stand for a long period of time, loss of strength, fatigue and fear of falling) as main barrier for participating in any sport activity. ${ }^{46}{ }^{54}$ The main cause reported for a change in sport activity was related to pain in the affected knee. ${ }^{46}$ Following KA, sports participation and activity level improved in comparison to the year before surgery, with activity levels returning eventually to a similar level as Pre-ORS. ${ }^{29} 3334363744-4648495152$ Patients who continued to participate in sport activities until close to surgery appear to become postoperatively even more active in low-impact and medium-impact sports than PreORS, and as equal or even more active than a healthy age-matched population sample. ${ }^{49556}$ In this respect, given the apparent relationship between preoperative and postoperative participation in sport activities, the lack of clinical, evidence-based guidelines regarding sport activities prior to surgery is remarkable.

It is known that there are complex factors that affect participation in physical activity before and after knee surgery, such as knee function, personal barriers and beliefs, self-efficacy, social support and ageing. ${ }^{18}$ Interestingly, among the perceived facilitators and barriers to exercise after surgery, reasons not related to the replaced knee are reported more frequently than those related to the replaced knee. ${ }^{27} 3337$ The most common facilitators to physical activity were related to the patients' motivation to improve symptoms or surgery outcomes, their personal commitment to physical activity and/ or to engage in an active lifestyle, and conscious monitoring and awareness of activity levels to ensure they were being active throughout the day. ${ }^{2733} 3754$ The most common reported barriers for participating in any sport activity after KA were a lack of motivation, precaution to preserve the prosthesis, a medical condition due to other comorbidities and pain or a physical limitation due to their knee. ${ }^{2733} 3753$ Notably, it appears that men are more likely to return to sport postoperatively than women. ${ }^{34} 44475051535557$ Among the measures of knee function, scores on the physical-related 36-Item Short Form Health Survey (SF-36) domains showed the strongest correlation with both number of sport activities ${ }^{28}$ and activity level. ${ }^{33}$ In this perspective, postoperative sport activities and activity levels appear to be more related to patient-specific factors rather than sociodemographic factors, type of surgery, implant, bearing surface diameter or operating surgeon. ${ }^{34}$

Based on the actual postoperative sport participation of patients as shown in this review and previous research, ${ }^{18}{ }^{58-61}$ the most popular sports were swimming, cycling and (Nordic) walking/hiking, ${ }^{27-31} \quad 33 \quad 37 \quad 46-53$ while participation in high-impact activities such as ball sports, running/jogging and skiing strongly decreased compared with Pre-ORS. After muscle strength and muscle control of the quadriceps and hamstring muscles have been sufficiently recovered, ${ }^{13}{ }^{19}$ it is encouraged to perform low-impact activities as they help improve general health and cardiovascular fitness. Muscular rehabilitation in terms of strength and coordination is in this sense important for the safety and protection of the joint, in which preoperative physiotherapy may be helpful to accelerate this recovery process postoperatively. ${ }^{62-64}$ Although it is typically advised to strongly discourage patients with knee arthroplasty from participation in highimpact sports, ${ }^{12} 1315$ 19-24 and a common sense of caution should be taken in mind, our findings do indicate that it is not impossible to participate in high-impact sport activities after KA. Notably, participation in (aqua)aerobics and fitness increased from Pre-ORS to Post-KA. During these medium-impact activities, it is recommended to put emphasis on a high number of repetitions with minimal resistance. $^{24}$

In terms of sport participation and improvement in UCLA score postoperatively, our results indicate that a UKA procedure achieves better outcomes in comparison to TKA. Nevertheless, it can be questioned whether a fair comparison can actually be made between UKA and TKA procedures in relation to sport participation and activity level. A selection bias may possibly be present as (younger) patients who are more motivated and/or have higher expectations already prior to the surgical procedure in regard to continued participation in athletic activities after KA are more likely to receive a UKA procedure. Motivation and expectation level both have been proven indeed to positively affect sport participation and activity levels after KA. ${ }^{35} 36$ As such, it can be argued that the reported differences in relation to sport and activity levels are a result of differences in motivation and expectation level of the involved patients groups already prior to the surgical procedure, rather than due to the used surgical procedure itself. This assumption is supported by our finding that patients with TKA who remained active until the period within 1 year prior to surgery showed a similar pattern as patients with UKA in terms of postsurgery sport participation.

A number of common methodological issues need to be taken into account for the interpretation of the presented outcomes of this systematic review. Due to the retrospective character of many studies, for example, sport participation questionnaires are prone to recall bias as many rely on a patient's ability to describe sporting activity several years before the study was carried out. Furthermore, many studies were faced with follow-up loss of more than 5\%. It can be argued that the participants whom were lost in follow-up are likely to be less satisfied with their results of the surgical procedures in comparison to the reported data in this systematic review, leading to a possible underestimation of the negative outcomes. 
Future studies are urged to take these potential biases into consideration when designing and performing their study. Finally, this review bears some limitations on itself that we would like to address, including the lack of an a priori registration of the review protocol and screening for inclusion by a single reviewer only. The authors acknowledge that screening for inclusion by a single reviewer lowers scientific rigour and enhances the potential risk of missing relevant literature. Despite the fact that the review protocol was set a priori by the authors and did not changed throughout the research process, a priori registration of the review protocol would have strengthened the study and is encouraged for future research.

\section{CONCLUSIONS}

$\mathrm{KA}$ is an effective treatment in relation to sports participation and activity level, with the potential to become as equal or even more active than healthy age-matched peers. Despite a decline in high-impact sports participation, our findings indicate that it is possible to return to similar levels of activity as Pre-ORS after both UKA and TKA. Patients who continued to participate in sport activities until close to surgery appear to become even more active in low-impact and medium-impact sports than PreORS. Most patients who stopped participating in sport activities prior to surgery, however, do not seem to reinitiate their sport activities after surgery. As such, to achieve the full benefits out of KA, clinical guidelines and strategies aimed to keep patients capable and motivated to participate in sport activities until close before and after surgery are warranted.

\section{Twitter Marco J Konings @MJ_Konings}

Contributors MJK and KC conceptualised the study. MJK conducted the literature searches, study selection, data extraction and study quality assessment. MJK drafted the initial version of the manuscript. All authors critically revised the draft manuscript and contributed to the subsequent revisions and the final version of the manuscript.

Funding The authors have not declared a specific grant for this research from any funding agency in the public, commercial or not-for-profit sectors.

Competing interests None declared.

Patient consent for publication Not required.

Provenance and peer review Not commissioned; externally peer reviewed.

Data availability statement Data are available upon request.

Open access This is an open access article distributed in accordance with the Creative Commons Attribution Non Commercial (CC BY-NC 4.0) license, which permits others to distribute, remix, adapt, build upon this work non-commercially, and license their derivative works on different terms, provided the original work is properly cited, appropriate credit is given, any changes made indicated, and the use is non-commercial. See: http://creativecommons.org/licenses/by-nc/4.0/.

\section{ORCID iD}

Marco J Konings http://orcid.org/0000-0003-0239-2020

\section{REFERENCES}

1 Lygre SHL, Espehaug B, Havelin LI, et al. Pain and function in patients after primary unicompartmental and total knee arthroplasty. J Bone Joint Surg Am 2010;92:2890-7.

2 Farquhar SJ, Reisman DS, Snyder-Mackler L. Persistence of altered movement patterns during a sit-to-stand task 1 year following unilateral total knee arthroplasty. Phys Ther 2008;88:567-79.
3 Pabinger $\mathrm{C}$, Lothaller $\mathrm{H}$, Geissler A. Utilization rates of kneearthroplasty in OECD countries. Osteoarthritis Cartilage 2015;23:1664-73.

4 Graves SE, Davidson D, Ingerson L, et al. The Australian orthopaedic association national joint replacement registry. Med J Aust 2004;180:S31-4.

5 Sabatini L, Giachino M, Risitano S, et al. Bicompartmental knee arthroplasty. Ann Transl Med 2016;4:5.

6 Wilson HA, Middleton R, Abram SGF, et al. Patient relevant outcomes of unicompartmental versus total knee replacement: systematic review and meta-analysis. BMJ 2019;364:I352.

7 Brown NM, Sheth NP, Davis K, et al. Total knee arthroplasty has higher postoperative morbidity than unicompartmental knee arthroplasty: a multicenter analysis. J Arthroplasty 2012;27:86-90.

8 De Vroey H, Staes F, Vereecke E, et al. Lower extremity gait kinematics outcomes after knee replacement demonstrate arthroplasty-specific differences between unicondylar and total knee arthroplasty: a pilot study. Gait Posture 2019;73:299-304.

9 Kleeblad LJ, van der List JP, Zuiderbaan HA, et al. Larger range of motion and increased return to activity, but higher revision rates following unicompartmental versus total knee arthroplasty in patients under 65: a systematic review. Knee Surg Sports Traumatol Arthrosc 2018;26:1811-22.

10 Arirachakaran A, Choowit P, Putananon C, et al. Is unicompartmental knee arthroplasty (UKA) superior to total knee arthroplasty (TKA)? A systematic review and meta-analysis of randomized controlled trial. Eur J Orthop Surg Traumatol 2015;25:799-806.

11 Webster KE, Wittwer JE, Feller JA. Quantitative gait analysis after medial unicompartmental knee arthroplasty for osteoarthritis. $J$ Arthroplasty 2003;18:751-9.

12 Jassim SS, Douglas SL, Haddad FS. Athletic activity after lower limb arthroplasty: a systematic review of current evidence. Bone Joint $J$ 2014;96-B:923-7.

13 Healy WL, Sharma S, Schwartz B, et al. Athletic activity after total joint arthroplasty. J Bone Joint Surg Am 2008;90:2245-52.

14 Bradbury N, Borton D, Spoo G, et al. Participation in sports after total knee replacement. Am J Sports Med 1998;26:530-5.

15 Golant A, Christoforou DC, Slover JD, et al. Athletic participation after hip and knee arthroplasty. Bull NYU Hosp Jt Dis 2010;68:76-83.

16 Jones DL. A public health perspective on physical activity after total hip or knee arthroplasty for osteoarthritis. Phys Sportsmed 2011;39:70-9.

17 Mora JC, Valencia WM. Exercise and older adults. Clin Geriatr Med 2018;34:145-62.

18 Barber-Westin SD, Noyes FR. Aerobic physical fitness and recreational sports participation after total knee arthroplasty. Sports Health 2016;8:553-60.

19 Healy WL, lorio R, Lemos MJ. Athletic activity after joint replacement. Am J Sports Med 2001;29:377-88.

20 Healy WL, lorio R, Lemos MJ. Athletic activity after total knee arthroplasty. Clin Orthop Relat Res 2000:65-71.

21 Kuster MS, Spalinger E, Blanksby BA, et al. Endurance sports after total knee replacement: a biomechanical investigation. Med Sci Sports Exerc 2000;32:721-4.

22 Laursen MK, Andersen JB, Andersen MM, et al. Danish surgeons allow the most athletic activities after total hip and knee replacement. Eur J Orthop Surg Traumatol 2014;24:1571-7.

23 Meester SB, Wagenmakers R, van den Akker-Scheek I, AkkerScheek vanden I, et al. Sport advice given by Dutch orthopaedic surgeons to patients after a total hip arthroplasty or total knee arthroplasty. PLoS One 2018;13:e0202494.

24 Dagneaux L, Bourlez J, Degeorge B, et al. Return to sport after total or unicompartmental knee arthroplasty. EFORT Open Rev 2017;2:496-501.

25 Jones DL, Cauley JA, Kriska AM, et al. Physical activity and risk of revision total knee arthroplasty in individuals with knee osteoarthritis: a matched case-control study. J Rheumatol 2004;31:1384-90.

26 Diduch DR, Insall JN, Scott WN, et al. Total knee replacement in young, active patients. long-term follow-up and functional outcome. J Bone Joint Surg Am 1997;79:575-82.

27 Pietschmann MF, Wohlleb L, Weber P, et al. Sports activities after medial unicompartmental knee arthroplasty Oxford III-what can we expect? Int Orthop 2013;37:31-7.

28 Naal FD, Fischer M, Preuss A, et al. Return to sports and recreational activity after unicompartmental knee arthroplasty. Am J Sports Med 2007;35:1688-95.

29 Fisher N, Agarwal M, Reuben SF, et al. Sporting and physical activity following Oxford medial unicompartmental knee arthroplasty. Knee 2006;13:296-300. 
30 Mayr HO, Reinhold M, Bernstein A, et al. Sports activity following total knee arthroplasty in patients older than 60 years. J Arthroplasty 2015;30:46-9.

31 Vielgut I, Leitner L, Kastner N, et al. Sports activity after low-contactstress total knee arthroplasty - a long term follow-up study. Sci Rep 2016;6:24630.

32 Marker DR, Mont MA, Seyler TM, et al. Does functional improvement following TKA correlate to increased sports activity? lowa Orthop J 2009;29:11-16.

33 Chang MJ, Kim SH, Kang YG, et al. Activity levels and participation in physical activities by Korean patients following total knee arthroplasty. BMC Musculoskelet Disord 2014;15:240.

34 Williams DH, Greidanus NV, Masri BA, et al. Predictors of participation in sports after hip and knee arthroplasty. Clin Orthop Relat Res 2012;470:555-61.

35 Bonnin M, Laurent JR, Parratte S, et al. Can patients really do sport after TKA? Knee Surg Sports Traumatol Arthrosc 2010;18:853-62.

36 Ponzio DY, Chiu Y-F, Salvatore A, et al. An analysis of the influence of physical activity level on total knee arthroplasty expectations, satisfaction, and outcomes: increased revision in active patients at five to ten years. J Bone Joint Surg Am 2018;100:1539-48.

37 Walker T, Gotterbarm T, Bruckner T, et al. Return to sports, recreational activity and patient-reported outcomes after lateral unicompartmental knee arthroplasty. Knee Surg Sports Traumatol Arthrosc 2015;23:3281-7.

38 Moher D, Liberati A, Tetzlaff J, et al. Preferred reporting items for systematic reviews and meta-analyses: the PRISMA statement. PLoS Med 2009;6:e1000097.

39 Ghomrawi HMK, Lee Y-Y, Herrero C, et al. A Crosswalk between UCLA and lower extremity activity scales. Clin Orthop Relat Res 2017;475:542-8

40 Brandes M, Ringling M, Winter C, et al. Changes in physical activity and health-related quality of life during the first year after total knee arthroplasty. Arthritis Care Res 2011;63:328-34.

41 Lee DC, Kim DH, Scott RD, et al. Intraoperative flexion against gravity as an indication of ultimate range of motion in individual cases after total knee arthroplasty. J Arthroplasty 1998;13:500-3.

42 Kennedy DM, Stratford PW, Riddle DL, et al. Assessing recovery and establishing prognosis following total knee arthroplasty. Phys Ther 2008;88:22-32.

43 Slim K, Nini E, Forestier D, et al. Methodological index for nonrandomized studies (MINORS): development and validation of a new instrument. ANZ J Surg 2003;73:712-6.

44 Scott CEH, Turnbull GS, Powell-Bowns MFR, et al. Activity levels and return to work after revision total hip and knee arthroplasty in patients under 65 years of age. Bone Joint J 2018;100-B:1043-53.

45 Scott CEH, Turnbull GS, MacDonald D, et al. Activity levels and return to work following total knee arthroplasty in patients under 65 years of age. Bone Joint J 2017;99-B:1037-46.

46 Panzram B, Bertlich I, Reiner T, et al. Cementless unicompartmental knee replacement allows early return to normal activity. BMC Musculoskelet Disord 2018;19:18.

47 Chatterji U, Ashworth MJ, Lewis PL, et al. Effect of total knee arthroplasty on recreational and sporting activity. ANZ J Surg 2005;75:405-8.
48 Canetti R, Batailler C, Bankhead C, et al. Faster return to sport after robotic-assisted lateral unicompartmental knee arthroplasty: a comparative study. Arch Orthop Trauma Surg 2018;138:1765-71.

49 Walker T, Streit J, Gotterbarm T, et al. Sports, physical activity and patient-reported outcomes after medial Unicompartmental knee arthroplasty in young patients. J Arthroplasty 2015;30:1911-6.

50 Hepperger C, Gföller P, Abermann E, et al. Sports activity is maintained or increased following total knee arthroplasty. Knee Surg Sports Traumatol Arthrosc 2018;26:1515-23.

51 Jahnke A, Mende JK, Maier GS, et al. Sports activities before and after medial unicompartmental knee arthroplasty using the new Heidelberg sports activity score. Int Orthop 2015;39:449-54.

52 Ho JC, Stitzlein RN, Green CJ, et al. Return to sports activity following UKA and TKA. J Knee Surg 2016;29:254-9.

53 Huch K, Müller KAC, Stürmer T, et al. Sports activities 5 years after total knee or hip arthroplasty: the Ulm osteoarthritis study. Ann Rheum Dis 2005;64:1715-20.

54 Pellegrini CA, Ledford G, Chang RW, et al. Understanding barriers and facilitators to healthy eating and physical activity from patients either before and after knee arthroplasty. Disabil Rehabil 2018;40:2004-10.

55 Smith TO, Mansfield M, Dainty J, et al. Does physical activity change following hip and knee replacement? matched case-control study evaluating physical activity scale for the elderly data from the osteoarthritis initiative. Physiotherapy 2018;104:80-90.

56 Meessen JMTA, Peter WF, Wolterbeek R, et al. Patients who underwent total hip or knee arthroplasty are more physically active than the general Dutch population. Rheumatol Int 2017;37:219-27

57 Wylde V, Livesey C, Blom AW. Restriction in participation in leisure activities after joint replacement: an exploratory study. Age Ageing 2012;41:246-9.

58 Witjes S, Gouttebarge V, Kuijer PPFM, et al. Return to sports and physical activity after total and Unicondylar knee arthroplasty: a systematic review and meta-analysis. Sports Med 2016;46:269-92.

59 Waldstein W, Kolbitsch P, Koller U, et al. Sport and physical activity following unicompartmental knee arthroplasty: a systematic review. Knee Surg Sports Traumatol Arthrosc 2017;25:717-28.

60 Arnold JB, Walters JL, Ferrar KE. Does physical activity increase after total hip or knee arthroplasty for osteoarthritis? A systematic review. J Orthop Sports Phys Ther 2016;46:431-42.

61 Papalia R, Del Buono A, Zampogna B, et al. Sport activity following joint arthroplasty: a systematic review. Br Med Bull 2012;101:81-103.

62 Moyer R, Ikert K, Long K, et al. The value of preoperative exercise and education for patients undergoing total hip and knee arthroplasty: a systematic review and meta-analysis. JBJS Rev 2017; 5:e2.

63 Kwok IHY, Paton B, Haddad FS. Does pre-operative physiotherapy improve outcomes in primary total knee arthroplasty? - a systematic review. J Arthroplasty 2015;30:1657-63.

64 Peer MA, Rush R, Gallacher PD, et al. Pre-surgery exercise and post-operative physical function of people undergoing knee replacement surgery: a systematic review and meta-analysis of randomized controlled trials. J Rehabil Med 2017;49:304-15. 